\title{
Endourological Management of Forgotten Double J Ureteral Stents: A Single Centre Study
}

\author{
Ankur Jhanwar*, Ankur Bansal, Gaurav Prakash and Satyanarayan Sankhwar \\ King George Medical University, India
}

Submission: December 26, 2017; Published: February 01, 2017

*Corresponding author: Ankur Bansal, Department of Urology, King George Medical College, Uttar Pradesh, India, Tel: +91-8853807160; Email: ankurbansaldmc@gmail.com

\begin{abstract}
Background: Double J (DJ) ureteral stent is being used in various urological and non-urological procedures. Indications for double J ureteric stents includes; to negotiate the obstruction and maintain adequate drainage from kidneys, help in localization and prevention of ureteral injuries during various gynecological and general surgical procedures and as an adjunctive procedure to extra corporeal shock wave lithotripsy (ESWL). A forgotten DJ stent is not uncommon in developing countries and is particularly seen in patients with poor socioeconomic status. These patients present with flank pain, hematuria, irritative voiding lower urinary tract symptoms (LUTS) and even lend up with features of renal failure. Management of forgotten DJ ureteral stents is not only important for patient's perspective but also for the surgeon's prospect due to its medico-legal implications.
\end{abstract}

Methods: Retrospective data search from 2008-2015 revealed 47 patients admitted with forgotten ureteral stent includes (broken and encrusted) were managed endoscopically. Patients were evaluated with X-ray, ultrasonography of Kidney ureter and bladder region (KUB), intravenous urography (IVU) and non-contrast computed tomography, renal function test (where indicated). Endourological procedure for DJ stent removal was decided according to the location of stents.

Results: A total of 47 patients (males: 34, females: 13) were included in the study. The mean age of patients was 23.53 years (range 4-65 years), mean duration of stent insertion was 39 months (4-68 months). Fifteen patients (31.9\%) had stent insertion following percutaneous nephrolithotomy, 8 (16.96\%) following ureteroscopic lithotripsy (URSL), 3 (6.3\%) following pyeloplasty, 10 (21.2\%) following ureterolithotomy, $6(12.72 \%)$ following pyelolithotomy and $3(6.3 \%)$ following ureteric re-implantation. Two patients (4.2\%) had DJ insertion for bilateral upper ureteric calculus with deranged renal function.

Conclusion: Forgotten double J ureteral stent still a common and preventable complication in developing nations, patients may lend up in renal failure. Its removal is a challenging task.

Keywords: Forgotten; Encrusted; Broken; Double J stents

Abbreviations: DJ: Double J; ESWL: Extra Corporeal Shock Wave Lithotripsy; LUTS: Lower Urinary Tract Symptoms; KUB: Kidney Ureter and Bladder Region; URSL: Ureteroscopic Lithotripsy; UTI: Urinary Tract Infection; PCNL: Percutaneous Nephrolithotomy; CLT: Cystolithotripsy

\section{Introduction}

Since its introduction in 1978 ureteral stents become an essential part of urologic practice [1]. Double J ureteral stent is being used in various urological and non -urological surgeries. Indications for double J ureteric stents placement is to negotiate the obstruction and maintain adequate drainage from kidneys, help in localization and prevention of ureteral injuries during various gynecological and general surgical procedures and as an adjunctive procedure to extra corporeal shock wave lithotripsy (ESWL) [2]. Double J stent insertion is also not without complications; various intra and post-operative complications related to double J stent have been mentioned in the literature. Management of forgotten double J ureteral stents is also important for the surgeon's prospect due to its medicolegal implications. This article highlights on unique complication of forgotten DJ stent and its endourological management and preventive measures to avoid devastating complications. To best of our knowledge, this is the first study of such kind which focused on etiology, endourological management and preventive measures of forgotten DJ stents.

\section{Materials and Methods}

A data of 47 patients (Male-34, Female-13) were reviewed and analyzed after ethical clearance from institutional review 
board in the department of Urology, King George's Medical University admitted with diagnosis of forgotten DJ stent (broken and encrusted) from 2008-2015. Forgotten Double J (DJ) stent was defined as the stent not removed or replaced within 6 months duration post operatively. Data collected includes, patient's and stent characteristics, cause for double J ureteral stent insertion, presenting symptoms, and reason for forgetting or avoiding stent removal on time and procedure of DJ stent removal. All patients were thoroughly evaluated for stent encrustation, fragmentation and location with plainfilm radiography, sometimes supplemented by intravenous urography ornon-contrast computed tomography (if serum creatinine was deranged). Ultrasonography of Kidney, ureter, bladder (KUB) was performed to assess the status of upper tract. Urine routine analysis with culture and sensitivity were done to rule out urinary tract infection (UTI). All patients were stabilized prior to intervention and preoperative antibiotics were administered according to culture report till repeat culture becomes sterile. Procedure performed for the removal of forgotten (broken or encrusted) double J stent were percutaneous nephrolithotomy (PCNL), combined PCNL and cystolithotripsy (CLT), Ureteroscopic lithotripsy (URSL), combined URSL and cystolithotripsy (CLT), and CLT alone. All procedures were performed under general or spinal anesthesia with standard technique. Holmium laser (Versa Power suite HO:YAG laser) and pneumatic lithoclast (Swiss Lithoclast 2, Wolf) were used for removal of hard encrustation over double J ureteral stents. Post-operatively stone clearance was evaluated with X-ray and ultrasonography of KUB region.

\section{Results}

Table 1: Baseline characteristics and reason for Double $\mathrm{J}$ stent insertion.

\begin{tabular}{|c|c|}
\hline No of Patients & 47 \\
\hline \multicolumn{2}{|c|}{ Gender } \\
\hline Male & 34 \\
\hline Female & 13 \\
\hline \multicolumn{2}{|c|}{ Mean Age (Years) } \\
\hline $\begin{array}{l}\text { Mean duration of stent } \\
\text { placement (months) }\end{array}$ & $39(4-68)$ \\
\hline Broken & $9(18.8 \%)$ \\
\hline Encrusted & $36976.32 \%)$ \\
\hline Procedure & (n) $\%$ \\
\hline PCNL & $1531.9 \%)$ \\
\hline URSL & $8(16.96 \%)$ \\
\hline Pyelolithotomy & $6(12.72 \%)$ \\
\hline Ureterolithotomy & $10(21.2 \%)$ \\
\hline Ureteric-re-implantation & $3(6.3 \%)$ \\
\hline Pyeloplasty & $3(6.3 \%)$ \\
\hline B/L DJ stenting & $2(4.2 \%)$ \\
\hline
\end{tabular}

A total of 47 patients (males-34, females-13) were included in the study. The mean age of patients was 23.53 years (range 4-65 years), mean duration of stent insertion was 39 months (4 -72 months). Fifteen patients (31.9\%) had stent insertion following percutaneous nephrolithotomy, 8 patients (16.96\%) following URSL for lower ureteric stone, 3 patients (6.3\%) following pyeloplasty, 10 patients $(21.2 \%)$ following ureterolithotomy, 8 patients (16.96\%) following pyelolithotomy, and 3 patients $(6.3 \%)$ following ureteric re-implantation (Table 1). PCNL was performed in 10 (21.2\%), combined PCNL and CLT in 15 (31.8\%), URSL in 12 (25.44\%), combined URSL and CLT in 18 (38.16\%), CLT alone in 11 (23.32\%) cases (Table 2). None of the 47 cases required open surgery or underwent extracorporeal shock wave lithotripsy (ESWL). Two patients (4.2\%) underwent hemodialysis prior to intervention to stabilize the condition. No major intra or post post-operative complication reported. (Figure $1 \& 2$ ).

Table 2: Patients presentation and procedure performed for removal of Double J stents.

\begin{tabular}{|c|c|}
\hline Presenting Symptoms & (n) $\%$ \\
\hline Flank pain & $23(48.7 \%)$ \\
\hline Irritative voiding LUTS & $18(38.16 \%)$ \\
\hline Hematuria & $4(8.48 \%)$ \\
\hline Renal failure & $2(4.24 \%)$ \\
\hline Procedure & $(\mathbf{n}) \%$ \\
\hline PCNL & $10(21.2 \%)$ \\
\hline PCNL + CLT & $15(31.8 \%)$ \\
\hline URSL & $12(25.44 \%)$ \\
\hline URS +CLT & $18(38.16 \%)$ \\
\hline CLT & $11(23.32 \%)$ \\
\hline
\end{tabular}

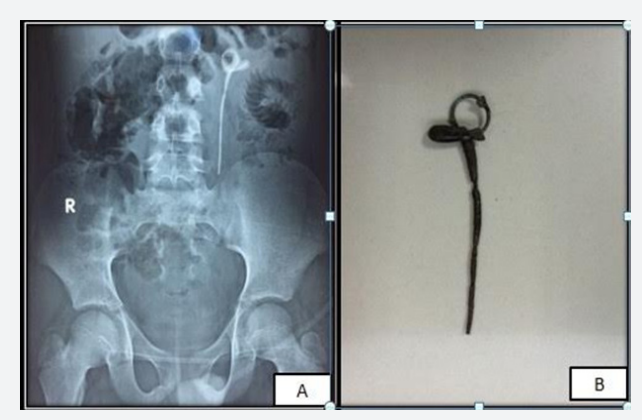

Figure 1 a: $\mathrm{X}$ ray KUB (Kidney, ureter and bladder) showing left forgotten JJ stent with encrustation and stone formation. b: Photograph of stent after removal. 


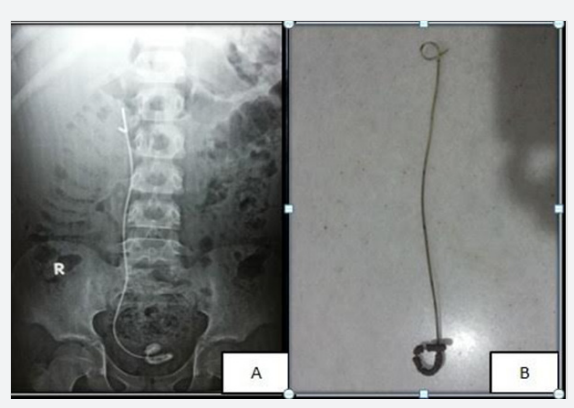

Figure 2 a: $X$ ray KUB (Kidney, ureter and bladder) showing right forgotten $\mathrm{JJ}$ stent with encrustation and stone formation. $\mathrm{b}$ : Photograph of stent after removal.

\section{Discussion}

Since its introduction in 1978, it is common in urologic and non-urologic practice. Multiple indications of Double J stent insertion described in the literature. Certain modifications made in double J stents to decrease stent related complications. Double J stent behave like a double edge sword, if left in situ for longer duration can cause significant morbidity. Various short term complications (3-9 weeks) related to stent include pain, frequency, dysuria and hematuria (stent syndrome). Long term complications include blockage, encrustation, fragmentation, up migration and hydronephrosis [3-6].

In this study, we observed that PCNL is the most common procedure performed (31.9\%) where double J stent is being used, followed by various other urological procedure both endoscopic and open. In this study, we observed multiple factors for forgotten double J stents. Patients were not informed properly about the stent insertion and its removal, which seems to be a major reason (38.16\%) (Table 2). Economic issue is also a major concern in developing nations, although hospital charges were low, but patient transportation to the tertiary health care centers for stent removal, as these facilities are not readily available at many centers and large volume of patients in government hospital increases the waiting period and cost of stent removal. Other important reasons were poor patient's compliance and low education status. The most common presenting symptom of patients with retained double J stents were flank pain in $23(48.7 \%)$, followed by irritative voiding lower urinary tract symptoms (LUTS) in 18 (38.16\%), hematuria in 4 (8.48\%) and 2 patients $(4.2 \%)$ included in the study were lend up in renal failure (Table 2). The reason being one patient had congenital solitary kidney and other had history of prior nephrectomy for benign pathology. Both these patients underwent hemodialysis before intervention. These complications of retained stents were also reported in literature. The management of forgotten ureteral stents is a challenging task due to encrustation which causes its removal very difficult and sometimes causes serious injury to the ureter. The required intervention depend on the site and condition of stent (broken or encrusted), function of the affected kidney and the availability of endourological instruments. Endourological management of a forgotten DJ stent is well established and there is algorithm for its use described by Somers in 1996. Multimodal treatment approach most commonly followed for DJ stent removal (7-10). Similarly, all forgotten double J stents were managed endoscopically without any intra or post-operative complications in present study. The most common treatment modality attempted for forgotten and retained DJ stents was combined URSL and CLT in (38.16\%) cases, followed by PCNL and CLT.

Certain precautions and guidelines must be ensured prior to double J insertion.

I. Patients and attendant should be well informed about the timely removal.

II. Patients details like age, name, telephone no. and address should be register in the hospital, so that patient can be timely informed for DJ stent removal.

III. If not contraindicated Check X ray KUB region should be performed and handed over to patients. So that he/ she remember about the stent in situ and its timely removal.

\section{Conclusion}

Forgotten Double J ureteral stent still a common complication in developing nations and its removal is a challenging task, patients may lend up in renal failure. The minimally invasive endoscopic procedures are preferred choice. Open surgery is an alternative option. However, best treatment is patient awareness and properly informed about the indwelling stents through proper follow up system.

\section{References}

1. Singh I, Indwelling JJ (2003) Ureteral stents: A current perspective and review of literature. J surg 65(5): 405-412.

2. Mattei A, Danuser H (2003) Stents in Urology. Ther Umsch 60(4): 233237.

3. Somers W (1996) Management of forgotten retained indwelling ureteral stents. Urology 47(3): 431-435.

4. Ringel A, ritcher S, shalev M, Nissenkon I (2000) Late complications of ureteral stents. EUR urol 38(1): 41-44.

5. Ivica S, Dragan S (2009) Long-term indwelling double-J stents: Bulky kidney and urinary bladder calculosis, spontaneous intra-peritoneal perforation of the kidney and peritonitis as a result of "forgotten" double-J stent. Vojnosanit Pregl 66(3): 242-244.

6. Kehinde EO, Al- Awadi KA, Tawheed A, Al-Hunayan A, Ali Y, et al. (2001) Factors affecting the fate of prolonged forgotten J Stents. Scand J Urol Nephrol 35(3): 222-227.

7. Tsai CC, Shen JT, Huang SP, Chang SF, Tsai LJ, et al. (2009) Use of holmium Laser to treat a forgotten double J stent with whole stent encrustation: a case report. Med Sci 25(10): 567-571.

8. Santin M, Motta A, Denyer SP, Cannas M (1999) Effect of the urine conditioning film on ureteral stent encrustation and characterization of its protein composition. Biomaterials 20(13): 1245-1251.

9. Rana AM, Sabooh A (2007) Management strategies and results for severely encrusted retained ureteral stents. J Endourol 21(6): 628-632.

10. Monga M, Klein E, Castãneda-Zúnniga WR, Thomas R (1995) The forgotten indwelling ureteral stent: a urological dilemma. Journal of Urology 153(6):1817-1819. 

(C) Co This work is licensed under Creative
Your next submission with Juniper Publishers will reach you the below assets

- Quality Editorial service

- Swift Peer Review

- Reprints availability

- E-prints Service

- Manuscript Podcast for convenient understanding

- Global attainment for your research

- Manuscript accessibility in different formats

( Pdf, E-pub, Full Text, Audio)

- Unceasing customer service

Track the below URL for one-step submission https://juniperpublishers.com/online-submission.php 